\title{
Facilitating an Active Transmit-only RFID System Through Receiver-based Processing
}

\author{
Yu Zhang, Gautam Bhanage, Wade Trappe, Yanyong Zhang, and Rich Howard \\ Wireless Information Network Laboratory \\ Rutgers University, Piscataway, NJ 08854, USA \\ $\{$ yu,gautamb,trappe,yyzhang,reh $\} @$ winlab.rutgers.edu
}

\begin{abstract}
Many asset tracking applications demand long-lived, low-cost, and continuous monitoring of a large number of items, which has posed a significant challenge to today's RFID design. In order to satisfy these requirements, we propose to adopt transmit-only tags without a receiver, which can offer both low power and low cost. In spite of their great potential, such a platform faces many challenges since it cannot sense the channel, causing the collisions among tag transmissions to be high. It is thus crucial to employ effective multi-user detection schemes at the tag reader to extract valid information from collided signals. Traditional detection schemes, such as successive cancelation, cannot be directly applied to the targeted system. Firstly, due to the simplicity of receiver-less transmit-only tags, there is no mechanism for feedback to the tags that is traditionally needed for accurate multi-user detection. More importantly, these schemes impose serious processing and memory requirements on the underlying system, which makes real-time tracking impossible. In this study, we address these challenges by performing a statistical estimation of the signal amplitude, and by dividing the received signal sequence (from all the tags) and assigning each block to one reader. We also adopt an online learning mechanism so that readers can anticipate the tags that belong to them. We show that the proposed detection algorithm can achieve low detection error under realistic system conditions.
\end{abstract}

\section{INTRODUCTION}

RFID technologies promise the ability to monitor a variety of assets [1-3]. Although RFID technologies have had many success stories, such as the EZ-Pass system for electronic toll collection, the ability for RFID systems to simultaneously monitor a large amount of items, such as would be needed for more tightly managing inventory, while also having low-cost, has continued to be a significant challenge. Ideally, in order for an enterprise to track its assets, it is desirable to identify precisely where individual items are at any moment over an extended period of time. Passive tags that depend on harvesting power from a basestation have performance bounded by the regulatory limits of costly high-power basestations (e.g. on the order of 4 Watts). Alternatively, at lower frequencies they work well, but only for short range $(\sim 1 \mathrm{~cm})$ sensing, which cannot provide continual tracking. Active tags overcome many of these limits and provide improved range and reliability. Unfortunately, the standard assumption that such tags would consume a large amount of power has made it impossible to continuously monitor over a period of years.

In order to avoid the shortcomings associated with both types of tags, in [4], we proposed to adopt transmit-only active tags, which have the long range of traditional active tags, but without their high power consumption. In a system built on such transmit-only tags, tags periodically announce their presence by sending out their tag IDs, and the processing burden is placed on the tag reader. Since transmit-only tags cannot sense the channel, their transmissions are likely to collide with each other, especially for a system with a large number of tags. Thus, the tag reader must employ effective multi-user detection schemes to extract tag IDs from collided signals. In our earlier work [4], we tested the feasibility of putting together such a system by using several simple detection schemes. While our results in [4] provided some initial support towards building a realistic tracking system using transmit-only tags, the detection accuracy left room for significant improvement, especially for a dense RFID system.

To address this need, in this study, we focus on the development of a specialized multi-user detection scheme suitable for transmit-only RFID systems. Our starting point is the popular successive cancelation algorithm. We formulate the successive cancelation algorithm in the context of our random on-off keyed tag signals, and discuss the algorithm for both coherent and non-coherent detection scenarios. As successive cancelation suffers from high computational and memory complexity, which is disadvantageous for real-time asset tracking, we then present an improved detection scheme that significantly reduces resource complexity while maintaining desirable detection performance. We then examine the performance of 
our tag detection scheme by evaluating it in the context of a broader system. For this system, we make each tag reader responsible for multiple tags, and discuss several approaches to achieve efficient tag handoff between readers.

The paper is organized as follows. First, we will examine related work in Section II. Then, in Section III, we introduce our basic RFID system and communication model. Next, in Section IV, we turn to the problem of identifying tags in spite of collisions by using a successive cancelation algorithm that has been customized for our RFID problem. In Section V, we support the feasibility of our approach by providing a scalability analysis, and also propose an algorithm for updating tag lists as tagged items move through the environment. An evaluation is provided in Section VI, and we conclude the paper in Section VII.

\section{RELATED WORK}

Recently, radio frequency identification has attracted significant research interest [2,3]. However, in order for RFID to succeed, it is necessary to have low-cost RFID systems that have good performance [3], and thus there has been extensive work to design a low cost tag [5]. One particularly difficult challenge that is faced by RFID tags is the issue of multiple tags transmitting at the same time. For contact-read tags, this issue is not serious, but for noncontact tags, the issue of identifying tags in spite of the potential for collisions is very significant. One approach to handling collisions is to employ a basic CSMA-style medium access control mechanism [6], or other MAC protocol, such as an ALOHA [7-11] strategy. More recently, for tags with receive capabilities, a tree based approach has been explored [12-16].

All of the above methods require the tags to have receive functionality, which inevitably increases the cost and power consumption of a tag. In this paper, we propose to completely remove the receiver from the tag. Rather, each tag transmits periodically, and we place the task of detection and collision resolution on the readers. This is similar to DS/CDMA $[17,18]$, which allows multiple users transmit simultaneously, and the receiver decodes transmissions via de-spreading. For such systems, there are many detection methods, such as the optimum receiver, MMSE receiver, and successive cancelation methods [18-21].

However, unlike conventional multi-user communications, we do not have a feedback mechanism for power control, as is used in [18-21]. Rather, in this work we estimate which tags are present using a modified successive cancelation method that estimates the amount of tags present as well as the received signal level at the base station. Recently, [4] introduced a method using derivatives of correlation functions to estimate the transmission times of tags in an RFID system. However, because of near-far problems, the derivative method does not perform well in some cases. To handle these problems, this paper improves upon traditional successive cancelation $[17,18,20,21]$ and the derivative method.

\section{SYSTEM MODEL}

\section{A. RFID System Model}

A typical RFID system is composed of four components: the tag, the reader, application software that makes use of the data at the reader, and a computer system that is connected by the reader.

Similarly, in our model, there are a number of tags. Each tag has a unique identifier of length $L$ (in this paper, we shall use $L=100 \delta$ for our discussions, where $\delta$ is the time taken to transmit one bit and thus serves as the unit). In general, a proper $L$ is chosen to ensure that the necessary number of tags is far less than $2^{L}$. We shall assume that the tags transmit their tag identifiers as beacons in order to support the detection of individual tags, and that the tags transmit periodically with a period of $T$ bits of transmission duration. In order to reduce the collisions, $T>>L$. On the other hand, $T$ should not be too large, so that real-time detection is guaranteed. A data rate of $1 \mathrm{Mb} / \mathrm{s}$ was chosen as this corresponds to the rate supported by the current generation of low-cost radio chips, giving $L=10^{-4} \mathrm{sec}$ and we used $T=1 \mathrm{sec}$ for the period.

In our paradigm, the communication is one-way and asynchronous, i.e. tags only transmit signals. This greatly simplifies the logic on board a tag, and thus a tag's cost can be significantly reduced. There are two meanings of asynchronization. First, there is no synchronization between the transmission of tags. Since we need the logic of tags to be simple, we can neither require all tags transmit simultaneously nor in a TDMA manner. Further, the communication between tags and the reader is without synchronization, which implies that, in order to detect each tag, we need to also estimate the transmission time of that tag.

The tag reader, which we shall often refer to as a basestation, consists of an RF frontend that downshifts the received waveform to baseband, performs A/D, and supplies an appropriately sampled waveform to a processor for the detection and identification of the tags. The processing of the sampled waveform could be performed on-board (if the reader has a sufficiently powerful DSP/FPGA), or can be performed off-board by a PC (e.g. samples may be 


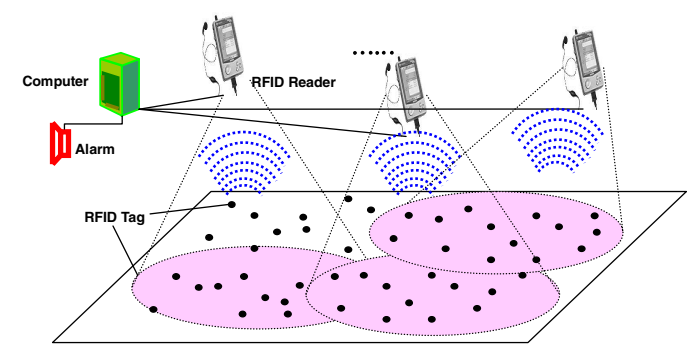

Fig. 1. RFID System Model.

transferred via PCI Express), as depicted in Figure 1. Our objective is to detect the tags in collisions without MAC or synchronization through received-based processing.

\section{B. RFID Communication Model}

For the sake of simplicity and cost-effectiveness, our tags use on-off keying (OOK) as the basic modulation scheme. This choice is motivated by our system implementation effort (which will be reported in a follow-on paper), where we have chosen a radio chip that uses OOK. For tag $i$, suppose that the transmitting baseband signal is $C_{i}(t)$, which is a randomly generated sequence composed of 1 or 0 . Here, $i \in\{1, \ldots, N\}$, represents the index of the tag, and $N$ is the total number of tags in the system. Since we use OOK modulation, given the carrier frequency $\omega_{c}$ and the phase $\phi_{i}$, the modulated signal will be $C_{i}(t) \cos \left(\omega_{c} t+\phi_{i}\right)$.

Suppose the distance of tag $i$ to the reader is $d_{i}$ and the starting time of the transmission is $\tau_{i}$, with $\tau_{i}$ being an integer multiple of the unit $\delta$. In a wireless fading environment, because the tag periodically transmits with period $T$ and $T>>L$, the received signal for tag $i$ is

$$
r_{i}(t)=\sum_{n=0}^{\infty} f_{i}\left(d_{i}\right) C_{i}\left(t-n T-\tau_{i}\right) \cos \left(\omega_{c} t+\phi_{i}\right)
$$

where $f_{i}\left(d_{i}\right)$ is the received amplitude resulting from path loss and the fading of tag $i$ 's signal.

Thus, the complete received signal is

$$
\begin{aligned}
r(t)= & \sum_{i=1}^{N} r_{i}(t)+n_{w}(t) \\
= & \sum_{i=1}^{N} \sum_{n=0}^{\infty} f_{i}\left(d_{i}\right) C_{i}\left(t-n T-\tau_{i}\right) \cos \left(\omega_{c} t+\phi_{i}\right) \\
& +n_{w}(t)
\end{aligned}
$$

At the demodulator, we first pass the received signal through a local oscillator, downshift and low-pass filter,

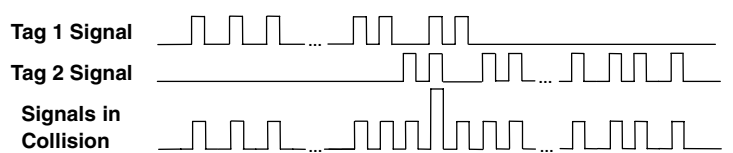

Fig. 2. Tag Signals in Collision.

to obtain the demodulated signal

$$
\begin{aligned}
R_{I}(t)= & L P\left\{r(t) \cos \left(\omega_{c} t+\widehat{\phi}\right)\right\} \\
= & \sum_{i=1}^{N} \sum_{n=0}^{\infty} f_{i}\left(d_{i}\right) C_{i}\left(t-n T-\tau_{i}\right) \cos \left(\phi_{i}-\widehat{\phi}\right) / 2 \\
& +n_{I}(t) \\
= & \sum_{i=1}^{N} \sum_{n=0}^{\infty} A_{I_{i}} C_{i}\left(t-n T-\tau_{i}\right)+n_{I}(t)
\end{aligned}
$$

where $n_{I}(t)$ is the $I$-phase filtered Gaussian noise and $A_{I_{i}}=f_{i}\left(d_{i}\right) \cos \left(\phi_{i}-\widehat{\phi}\right) / 2$. Similarly, we can get the $Q$-phase received signal:

$$
\begin{aligned}
R_{Q}(t)= & -L P\left\{r(t) \sin \left(\omega_{c} t+\widehat{\phi}\right)\right\} \\
= & \sum_{i=1}^{N} \sum_{n=0}^{\infty} f_{i}\left(d_{i}\right) C_{i}\left(t-n T-\tau_{i}\right) \sin \left(\phi_{i}-\widehat{\phi}\right) / 2 \\
& +n_{Q}(t) \\
= & \sum_{i=1}^{N} \sum_{n=0}^{\infty} A_{Q_{i}} C_{i}\left(t-n T-\tau_{i}\right)+n_{Q}(t)
\end{aligned}
$$

where $n_{Q}(t)$ is the $Q$-phase filtered Gaussian noise and $A_{Q_{i}}=f_{i}\left(d_{i}\right) \sin \left(\phi_{i}-\widehat{\phi}\right) / 2$.

\section{Detection Algorithm}

Due to collisions, the received signal at the reader during any time interval is the composition of one or more tag signals, as shown in (3). These signals may mask or compromise each other and thus corrupt the detection process. In this section, we explain our strategy and our algorithms to solve the detection problem.

\section{A. Detection Strategy}

Since the transmission times of these tags are not known, and the received signal at any time may be just a corrupted signal, we will focus on a period $T$ of the received signal as the input for processing. Because tags' transmissions are periodic, any received signal of duration $T$ contains a complete description of the information needed for detection.

In order to detect the $N$ RFID tags, we estimate the signal amplitude for each of the $N$ tags, and decide whether 
the estimation is valid. Here, we use a predefined minimum received signal strength of Threshold and declare the tag is present if the estimated signal strength is no smaller than Threshold. If the estimation is valid, we declare that the corresponding tag is present.

Suppose tags transmit independently, for tag $j$, the estimation of its received signal amplitude just depends on the received signal at the duration $\left[\tau_{j}, \tau_{j}+L\right)$. Thus, before the estimation of the signal amplitude, the transmission time of each tag must be estimated. The baseband tag signal is composed of $L$ pulses, each corresponding to either a 0 or 1 . For analysis, we assume the pulse $p(t)$ has duration $\delta$, with $\int_{0}^{\delta} p(\tau) d \tau=\int_{0}^{\delta} p^{2}(\tau) d \tau=1$, and represents a 1 . Similarly, a pulse $g(t)$, with the same duration and $\int_{0}^{\delta} g(\tau) d \tau=\int_{0}^{\delta} g^{2}(\tau) d \tau=1$ represents a 0 . It is straightforward to show that the average autocorrelation of each tag signal is $L / 2$, and that the crosscorrelation of tag signals is $L / 4$. Therefore, we can perform a correlation between the received signal of duration $T$ with each tag signal $C_{j}$ of duration $L$. The position of the peak in the correlated signal is the estimated transmission time $\hat{\tau_{j}}$. For $0 \leq t<T$, the correlated signal is

$$
\begin{aligned}
\rho_{j}(t)= & \int_{t}^{t+L} \sum_{i=1}^{N} A_{i} C_{i}\left(\tau-\tau_{i}\right) C_{j}(\tau-t) d \tau \\
& +\int_{t}^{t+L} C_{j}(\tau-t) n(\tau) d \tau .
\end{aligned}
$$

However, due to collisions, the estimation $\hat{\tau}_{j}$ may not be accurate, and the estimate only deteriorates further because of near-far issues. A natural solution is to estimate the tag with the maximum signal strength received at the reader, because it is most robust to collisions, and subtract its contribution from the received signal. By repeating this process, as long as the estimation of the received signal strength of each tag is sufficiently accurate, we remove large high-confidence components and amplify the presence of less powerful tags for further processing- a process known as successive cancelation [18].

\section{B. Coherent Detection}

For analytical simplicity, we first examine a coherent scheme. However, because coherent detection would require an increase in the cost of tags, we will not use it in our system. In coherent detection, we know the carrier phase of each tag. For simplicity, we do not align the phase $\hat{\phi}$ of the generated signal from the oscillator to the carrier phase $\phi_{i}$. The phase information is used only at the later validation. Suppose the recovered baseband received signal from the I-phase and Q-phase component

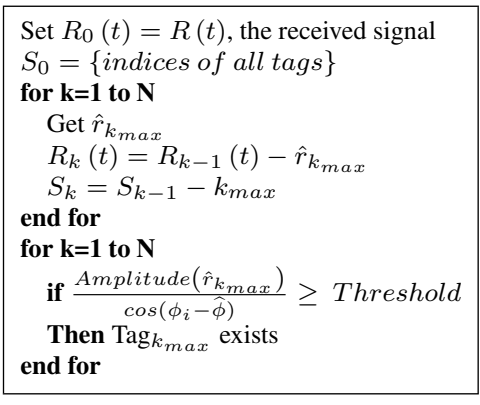

Fig. 3. Successive Cancelation Method for Coherent Detection

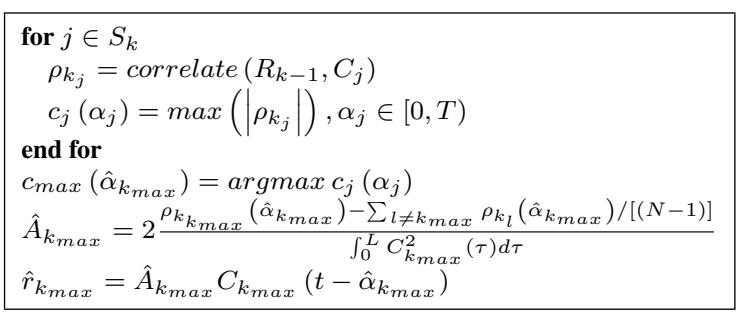

Fig. 4. Estimate of $r_{k_{\max }}$ from the residual signal $R_{k-1}(t)$.

is $R(t)=\sum_{i=1}^{N} \sum_{n=0}^{\infty} A_{i} C_{i}\left(t-\tau_{i}\right)+n(t)$, which is composed of $N$ tag signals, with a demodulated signal strength of $A_{i}$. Successive cancelation finds the tag signal with the maximum signal strength in each step, and subtracts this signal from the received signal, until all the tag signals are found, as shown in Fig.3. $S$ is the set which contains the index of undetected tags. At the first step, $S_{0}$ will consist of all tags indices. After each iteration, the index of the detected tag will be removed from the set.

Fig. 4 shows the estimation of both the transmission time and tag signal for the $k$ th round. First, we get the maximum value $c_{j}\left(\alpha_{j}\right)$ of the absolute value of the correlated signal $\rho_{k_{j}}$, where $j \in S_{k-1}$. We use absolute value because $A_{i}$ may be negative due to the phase offset. Next, the maximum value $c_{\max }\left(\hat{\alpha}_{k_{\max }}\right)$ among all $c_{j}\left(\alpha_{j}\right)$ is obtained, which is the maximum value of all the correlations at the $k$ th step. Then, $\hat{\alpha}_{k_{\max }}$ is the corresponding estimation of the transmission time of tag $k_{\max }$, and is the position of the maximum value at the correlated signal $\rho_{k_{k_{\max }}}$. For notational convenience, we assume $j=k_{\max }$.

In a correct detection, $\hat{\alpha}_{j}=\tau_{j}$. Without considering the noise,

$$
\begin{aligned}
\rho_{k_{j}}\left(\tau_{j}\right)= & \int_{\tau_{j}}^{\tau_{j}+L} A_{j} C_{j}^{2}\left(\tau-\tau_{j}\right) d \tau \\
& +\sum_{\substack{\left|\tau_{i}-\tau_{j}\right|<L \\
i \neq j}} \int_{\tau_{j}}^{\tau_{j}+L} A_{i} C_{i}\left(\tau-\tau_{i}\right) C_{j}\left(\tau-\tau_{j}\right) d \tau
\end{aligned}
$$


For other $l \neq j$,

$$
\begin{aligned}
\sum_{l \neq j} \rho_{k_{j}}\left(\tau_{j}\right)= & \sum_{\left|\tau_{i}-\tau_{j}\right|<L} \int_{\tau_{j}}^{\tau_{j}+L} A_{i} C_{i}\left(\tau-\tau_{i}\right) \\
& \cdot \sum_{l \neq k_{\max }} C_{l}\left(\tau-\tau_{j}\right) d \tau
\end{aligned}
$$

Since each $C_{l}$ is a random sequence of 0 or 1 , and as $N$ is big, according to law of large numbers, each bit of the sequence $\sum_{l \neq j} C_{l}\left(\tau-\tau_{j}\right)$ has roughly value $(N-1) / 2$. Thus, $\sum_{l \neq j} \rho_{k_{l}}\left(\tau_{j}\right) /[(N-1)]=$ $\sum_{\left|\tau_{i}-\tau_{j}\right|<L} \int_{\tau_{j}}^{\tau_{j}+L} A_{i} C_{i}\left(\tau-\tau_{i}\right) / 2 d \tau$. In addition, we know $E\left(\int_{\tau_{j}}^{\tau_{j}+L} A_{i} C_{i}\left(\tau-\tau_{i}\right) C_{l}\left(\tau-\tau_{j}\right) d \tau\right)$ is equal to $E\left(\int_{\tau_{j}}^{\tau_{j}+L} A_{i} C_{i}\left(\tau-\tau_{i}\right) d \tau\right) / 2$.

Then, we can get

$$
\begin{aligned}
E\left(\rho_{k_{j}}\left(\tau_{j}\right)\right)= & \int_{\tau_{j}}^{\tau_{j}+L} A_{i} C_{j}^{2}\left(\tau-\tau_{j}\right) / 2 d \tau \\
& +\sum_{l \neq j} \rho_{l_{i}}\left(\tau_{j}\right) /[(N-1)]
\end{aligned}
$$

Because every basis has similar weight, and each position is uniformly distributed with 1 or 0 , we have

$$
\hat{A}_{j} \approx 2 \frac{\rho_{k_{j}}\left(\tau_{j}\right)-\sum_{l \neq j} \rho_{l_{i}}\left(\tau_{j}\right) /[(N-1)]}{\int_{0}^{L} C_{j}^{2}(\tau) d \tau} .
$$

Therefore, $\hat{r}_{j}=\hat{A}_{j} C_{j}\left(t-\tau_{j}\right)$. As shown in Fig. 4, there is an estimation of $\hat{A}_{k_{\max }}$ for every cancelation. For coherent detection, we can set a threshold, and a tag is declared to be found if $\hat{A}_{k_{\max }} / \cos \left(\phi_{i}-\widehat{\phi}\right) \geq$ Threshold.

\section{Noncoherent Detection}

Now we extend the coherent scheme to handle the more general, noncoherent case where we do not know the carrier phase of each tag. Since the carrier phase of tags are randomly distributed, the contribution of some tags may be very small for either I-phase or Q-phase received signal, which complicates the near-far effect. Further, for non-coherent detection, because we don't know each tag's carrier phase, it is hard to set a proper Threshold to validate a correct estimation.

However, we note that typically, if the I-phase tag signal is small its Q-phase signal is large (and vice versa), due to the complementary properties of the trigonometric functions. This allows us to perform successive cancelation separately for both I-phase and Q-phase signals.

The algorithm is summarized in Fig. 5. For each channel of detection, the estimation is similar to the coherent

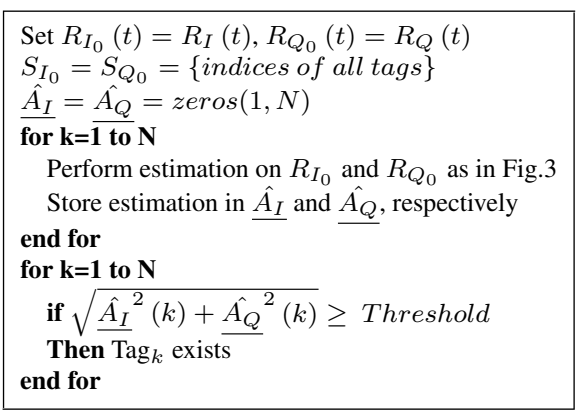

Fig. 5. Successive Cancelation Method for Noncoherent Detection

case. However, after we get the estimation of $\hat{A_{I_{i}}}$ and $\hat{A_{Q_{i}}}$, for $i=1, \ldots, N$, we declare we find $\operatorname{tag}_{i}$ as long as $\sqrt{{\hat{A_{i}}}^{2}(i)+\hat{A_{Q_{i}}}{ }^{2}(i)} \geq$ Threshold. By making use of both results, the final detection and estimation will be more accurate and complete. Further, the phase information is no longer important to the validation.

Naturally, we may get two different estimations of transmission times ${\hat{\tau_{i}}}_{i}$ and $\hat{\tau}_{Q_{i}}$ for $\operatorname{tag}_{i}$. We believe a large $\hat{A}$ will give a more accurate $\hat{\tau}$. Therefore, we decide $\hat{\tau_{i}}=\hat{\tau_{I_{i}}}$ if $\hat{A_{I_{i}}} \geq \hat{A_{Q_{i}}}$, otherwise, $\hat{\tau}_{i}=\tau_{Q_{i}}$. Further, if either $\hat{A_{I_{i}}}$ or $\hat{A_{Q_{i}}}$ is small and inaccurate, the implications are minimal. In addition, we can set Threshold slightly smaller than the minimum received signal strength to allow some fault tolerance. For the remainder of the paper, we restrict our discussion to non-coherent detection.

\section{Overlap Reduced Successive Cancelation Method}

A disadvantage of successive cancelation is its intensive computational and memory requirements. Further, the computational load doubles for non-coherent detection. We now propose an algorithm which can reduce the cost of computation by dividing the long received signal into contiguous overlapping blocks of manageable length, say $T_{s}$ samples, then performing successive cancelations for each block. On average, since the transmission time of all tags are randomly distributed with $[0, T)$, we can detect $N T_{s} / T$ tags for each block. Thus, at the second block, we need only perform correlation for $N-N T_{s} / T$ tags. As for the last block, only $N T_{s} / T$ correlations need to be performed. For example, if $T=10^{6}, T_{s}=10^{4}$, the computation can be reduced by about 2 . Since there might exist tags at the boundaries of each block, the neighboring blocks needs to overlap each other by a length of $L$, in order to detect every tag.

In order to keep the correlated values of tags for direct successive cancelation method, we need $4 N T$ bytes of memory if every correlated value needs four bytes. However, for our overlap reduced successive cancelation method, since we perform detection block-by-block, the 


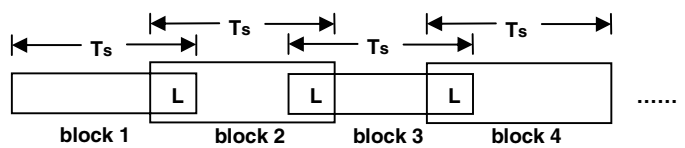

Fig. 6. Overlap Reduce Successive Cancelation Method.

necessary memory to store the correlated values is only $4 N T_{s}$ bytes. Thus, the relative necessary memory of overlap method over the conventional method is $T_{s} / T$. For example, if $T=10^{6}, T_{s}=10^{4}$, the memory needed is reduced by a factor of 100 .

\section{Scalability AnALYSis}

We now consider a general asset tracking application with a couple of thousand tags in a fairly spread out environment. Detection of all tags at a single receiver in such a scenario based on multi-user detection (MUD) will be extremely computationally intensive. A single receiver deployed in such an environment will also experience the near-far effect due to the large area covered.

In considering scaling, we will look at a deployment, as in Fig. 1, where the networked basestations are mounted in the ceiling and spread out over the volume to be monitored. The density of basestations is chosen to guarantee each tag can be loudly heard by at least one reader, and the number of tags per basestation is within the tolerance bounds with our algorithm. We believe such a basestation can be manufactured at a cost low enough to make this architecture attractive.

Each basestation ensures that it is dealing with a limited subset of tags from the universe of tags in the environment by maintaining a tag list $L_{i}$, where $i=1, \ldots, M$ and $M$ is the number of readers. Reader $_{i}$ is only responsible for the tags present in its tag list $L_{i}$. As long as each tag is properly taken care of by one or more readers, the total detection is complete and accurate. During operation, we define $\underline{L_{i}}=\left\{i_{1}, \cdots, i_{j}, \cdots\right\}$ as the list of tags that reader ${ }_{i}$ detected in one or more rounds of detection. In addition, the estimation of signal strength of each tag from reader ${ }_{i}$ is kept in $\underline{\hat{A}_{i}}=\left\{\left|\hat{A}_{i_{1}}\right|, \cdots,\left|\hat{A}_{i_{j}}\right|, \cdots\right\}$. These lists are initialized during system setup by having each reader check for all possible tags in the environment using an initialization algorithm and then switches to a higher speed operational algorithm afterwards.

\section{A. Initializing Phase and Online Phase}

The process is composed of two phases. In the initialization phase, readers don't know the distribution of the tags, and thus they have to scan all the tags. If a tag is

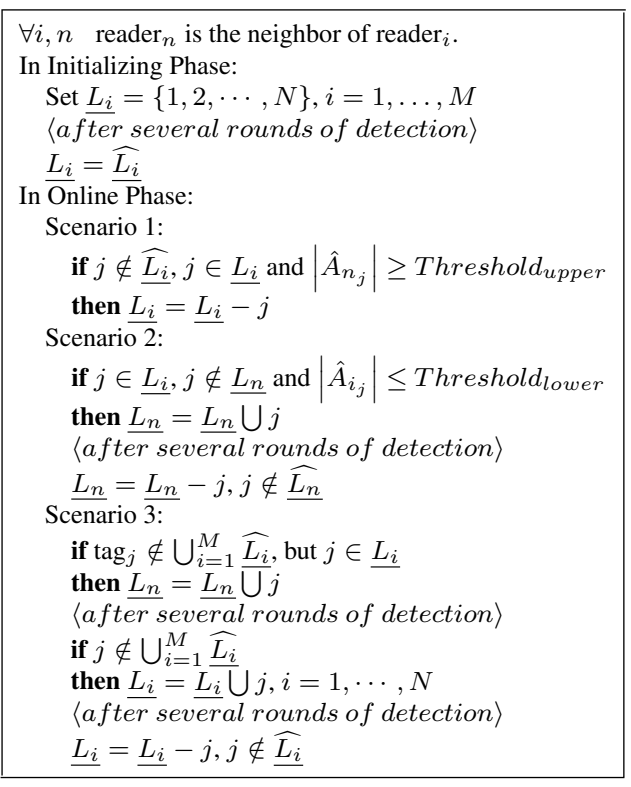

Fig. 7. Update of Tag List

detected by a reader, we say it is under this reader's coverage. A tag which is covered by multiple readers is called a boundary tag. In our simple propagation model, these tags will be at the boundary between the coverage areas shown in Fig. 1. For more complex environments, the geographical relation between the tags will not be as simple. Each reader keeps the tags that it covers in its tag list. In the initializing phase, we may run the detection for several rounds, to guarantee the tag list keeps complete information. The subsequent detections are all called second phase, or the online phase. In the second phase, each reader only needs to track the tags in its tag list.

\section{B. Soft Handoff and Update of Tag List}

The capability of the system to seamlessly monitor the movement of these tags in an integrated environment depends on the soft-handoff ability of our system. Periodic updates of the tag lists at each of the readers ensures a proper configuration of tag lists in realtime and makes systematic tracking of the tags feasible with low computation. Fig. 7 gives an overview of our distributed tag list update algorithm.

For initialization, we let each reader's tag list $\underline{L_{i}}$ contain all the tags. After several rounds of detections, each reader records the tags that it can detect in $\widehat{L}_{i}$, and then sets $\underline{L_{i}}=\widehat{L}_{i}$. In the online phase, there are three cases. The first case is that a tag loses the coverage of some readers. Meanwhile, the estimated signal strengths from that tag at the neighbors of these readers are above Threshold upper $_{\text {. }}$ Hence, we know it is a boundary tag that has moved and remove its index from those readers' tag lists. 
The second case happens if the estimated signal strength from a tag previously covered by only one reader

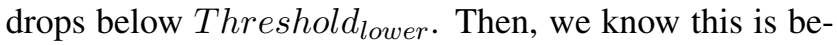
cause this tag has moved away from this reader. The surrounding readers will add this tag into its tag list. Over the next few rounds of detection, these readers will find out whether this tag is under their coverage. Those readers which don't cover it will remove it from its tag list.

The third case is that the system loses one tag. This may be because it has moved too fast or may be due to environmental interference. Since we know which readers last detected this tag, we add this tag into the neighbor readers' tag lists. After several rounds of detection, if we are still unable to find the tag, we add this tag into the tag lists of all the readers and remove the tag from the tag lists of the readers which don't cover the tag. We call this procedure detection compensation.

\section{Simulation}

Our simulations aim to study the effect of varying tag densities, near-far situations and signal-to-noise ratio on the overall detection performance. The detection performance of the system is measured in terms of probability of wrong detection of a tag and the mean squared error $(\epsilon)$ of the system in the amplitude estimation. Tag error rate is defined as the ratio of tags whose estimated transmission times do not conform to the actual ones. Suppose each tag has a correct amplitude $A_{i}$ of demodulated baseband signal at the receiver, while the amplitude estimate is $\hat{A}_{i}$, then the mean square error is $\epsilon=\sum_{i=1}^{N}\left(A_{i}-\hat{A}_{i}\right)^{2} / N$. The $\epsilon$ parameter indicates the ability of the estimation of the amplitudes of tag signals and the tag probability of wrong detection describes the probability of a detection failure with an arbitrary packet. Though these two metrics are correlated, the information conveyed by these parameters is individually significant. The simulations compare the relative performance of four detection algorithms: Correlation with first and second order derivative based post processing [4], traditional successive interference cancelation scheme $[20,22]$ and our improved successive cancelation algorithm.

\section{A. Simulation Setup}

Our simulation environment consists of 100 tags with each tag broadcasting a 100 bit sequence in a $10^{4} \mu \mathrm{s}$ burst. The average channel utilization corresponds to $10^{4}$ tags broadcasting with a period $1 s$. The start time for the $T=10^{4} \mu \mathrm{s}$ burst is randomly chosen by each tag. We assume the receiver correctly demodulates and uses a sufficient sampling rate, and thus our analysis is for baseband signals only.

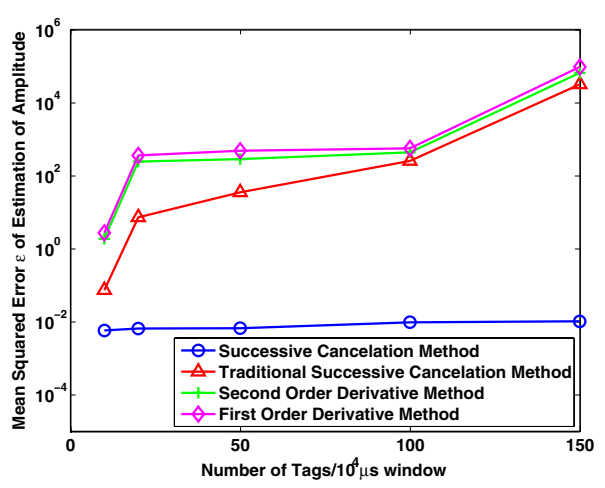

Fig. 8. The Mean Square Error of Estimation of the Amplitude versus the Number of Tags per Reader, $d_{\max }=d_{\min }$ and noise is 0 .

The physical layout of the experiment matches Fig. 1 where the tags are randomly distributed in a square plane, and the networked basestations are positioned above this plane. The minimum distance between tags and basestation is $d_{\min }$, and the maximum distance is $d_{\max }$. Periodically, each tag sends its tag ID signal with a transmission time uniformly distributed in the range $[0, T)$. We employ a free space path loss model, where the relationship between the received signal strength $P_{r}$ and the transmitting power $P_{t}$ is $P_{r}=\left(\sqrt{G_{l}} \lambda /(4 \pi d)\right)^{2} P_{t}$ [23], for $G_{l}$, $\pi$, and $\lambda$ are constants, and $d$ is the distance between the transmitter and the receiver.

\section{B. Effect Of Tag Collisions}

To study the effect of collisions on the detection scheme, we vary the number of collisions in the system by varying the number of tag transmissions from 10 to 150 in a single burst of $10^{4} \mu \mathrm{s}$. Other parameters are set as $d_{\min }=d_{\max }$, and ambient noise in the system is set to 0 . From Fig. 8 and Fig. 9, we see that generally the detection accuracy deteriorates as more collisions occur, for both the estimation of the transmission times and the amplitude. Due to the fact that tag signals will have varying levels of crosscorrelation, the overlapped transmission of multiple tags can affect detection performance. In the plots, this is evident for the derivative methods of [4], but we note that successive interference cancelation has better results than the other approaches. The reason is that successive cancelation method deducts the loudest signal in each cancelation, thereby alleviating the collisions among tags. Moreover, since we use a statistical method to cancel the effect of cross correlations, our successive cancelation is superior to the traditional approach.

\section{Near Far Effects}

Large variations of the path loss experienced by the transmission of different tags will affect the detection ac- 


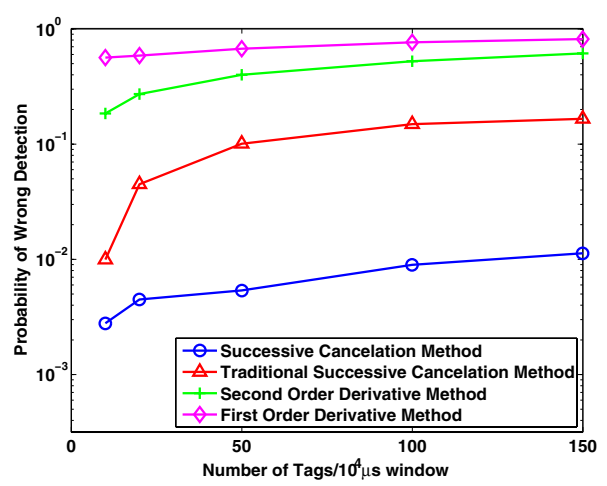

Fig. 9. The Tag Error Rate with Increasing Number of Tags per Reader, $d_{\max }=d_{\min }$ and noise is 0 .

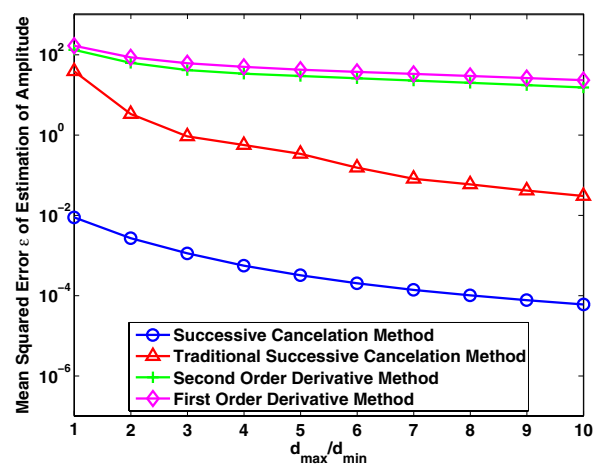

Fig. 10. The Mean Square Error of Estimation of the Amplitude versus $d_{\max } / d_{\min }$, the Ratio of Maximum Distance to Minimum Distance between AP and Tags, with $N=100$ in $10^{4} \mu s$ Window and noise is 0 .

curacy of our algorithm. To study this effect we consider a scenario with 100 tags in the environment, the noise levels fixed at 0 , and vary the $d_{\max } / d_{\min }$ from 1 to 10 to test the consequences of the near far effect.

Fig. 11 shows that successive cancelation has a better performance than the derivative based post processing method. Elimination of the loudest tags in consecutive estimations by the successive cancelation approach enables it to assuage the effect of the loud tags on the detection thereby allowing the transmission of the soft tags to stand out in the residue of the received signal. Our successive cancelation approach also manages to outperform the conventional successive cancelation scheme since the estimation approach used with our algorithm is better suited to solve our problem than the generic one. The signal strength of tags signals are generally smaller as $d_{\max } / d_{\min }$ increases, and the mean square error automatically decreases as shown in Fig. 10. We can see that mean square errors of successive cancelation methods decrease rapidly, which again supports its ability to handle near-far effects.

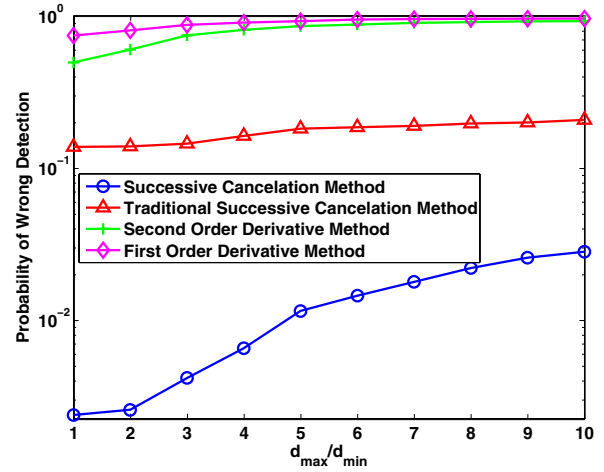

Fig. 11. The Tag Error Rate versus $d_{\max } / d_{\min }$, the Ratio of Maximum Distance to Minimum Distance between AP and Tags, with $N=100$ in $10^{4} \mu s$ Window and noise is 0 .

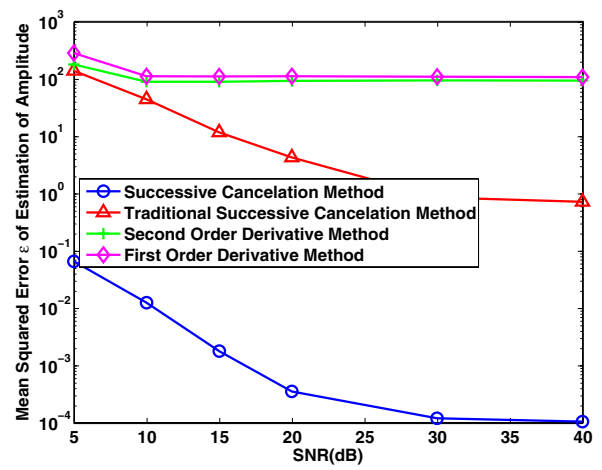

Fig. 12. The Mean Square Error of Estimation of the Amplitude versus $\operatorname{SNR}(\mathrm{dB})$, with $d_{\max } / d_{\min }=5$ and $N=100$ in $10^{4} \mu s$ Window.

\section{Noise Effects}

The presence of noise on the channel will bias the received signal and thus produce estimation errors with the time and amplitude estimations. To explicitly study the effect of noise on the detection accuracy, simulations were done with 100 tags in a $10^{4} \mu \mathrm{s}$ transmission burst. The SNR was varied from 40 to $5 \mathrm{~dB}$. To emphasize the effect of noise the $d_{\max } / d_{\min }$ ratio was set at 5 .

Fig. 12 plots the mean square error of the estimated amplitude as a function of the signal to noise ratio for the tag transmissions. Results show that the estimation error with our modified successive cancelation approach is far less than the other three. Since the other three methods do not attempt to make an accurate estimate of the amplitude of the received signal, the estimation error of these approaches with no power control is significant. Fig. 13 shows the probability of tag error for the same experiment. The plot shows that as the signal strength improves, the performance of our algorithm improves significantly as compared to the others due to better tag amplitude and transmission time estimation. From Fig. 12, we can see that our method overall has best performance among the 


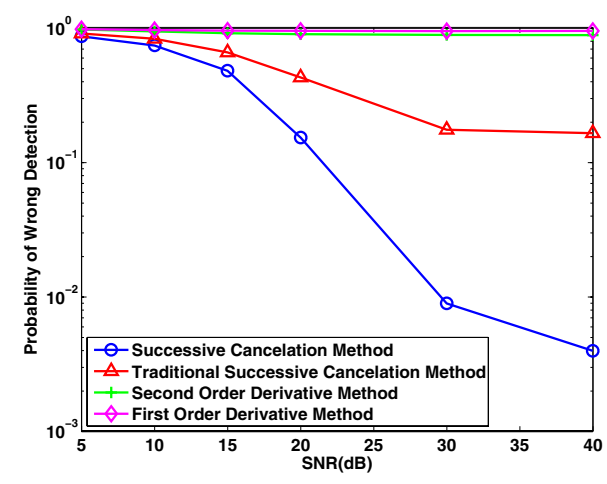

Fig. 13. The Tag Error Rate versus SNR(dB), with $d_{\max } / d_{\min }=5$ and $N=100$ in $10^{4} \mu s$ Window.

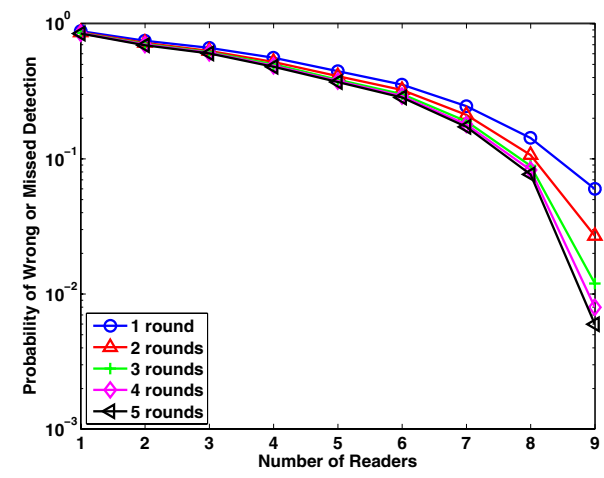

Fig. 14. Detection Rate in the Initializing Phase, with $d_{\max } / d_{\min }=$ $10, N=100$ and noise is $15 \mathrm{~dB}$.

four algorithms. The performance deteriorates as SNR decreases since the estimation of a tag's signal power degrades as tag power levels approach the noise level.

\section{E. Scalability Test}

The use of multiple readers in an integrated environment reduces the near-far effect thereby allowing for an improved detection accuracy with no power control mechanism. The goal of this experiment is to test the effect of varying reader count and using multiple detection rounds on a set of 100 tags transmitting over a $10^{4} \mu s$ burst. Since this experiment aims at testing the performance in a real environment we are considering a worst case distance ratio with the $d_{\max } / d_{\min }=10$. We consider the transmissions from the tags to be at a minimum $15 \mathrm{~dB}$ SNR.

Fig. 14 plots the improvement in the detection performance with various amounts of readers, and detection performed over multiple rounds. A tag is detected if it is successfully identified by at least one of the readers in at least one of the multiple rounds. The initial reading with 1 reader and a single round of detection shows a particularly poor performance because of the exceptionally harsh conditions chosen our simulations. Even under these conditions, it can be observed that as the number of readers

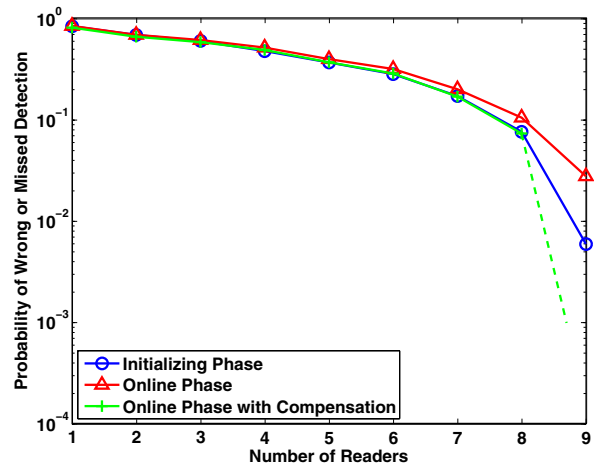

Fig. 15. Detection Rate Compared between Initializing Phase, Online Phase and Online Phase with Compensation, with $d_{\max } / d_{\min }=10$, $N=100$ in $10^{4} \mu s$ Window and SNR is $15 \mathrm{~dB}$. Please note that the dotted line was added to represent a number close to zero on a log scale plot.

and the rounds increase there is a considerable improvement in detection accuracy.

Fig. 15 tests the non-coherent successive interference cancelation scheme versus the number of readers at different phases of operation. The online phase shows the performance of the detection scheme when each basestations is responsible for all the tags in the environment. To scale computation, the online phase optimizes the tag list at each basestation. However, it may be observed that excessive truncation of the tag list results in missing some important collision information, which degrades performance. To correct this, the online phase with compensation ensures that the tag lists are properly updated to deal with the correct set of tags. The corresponding improvement results in a near accurate detection even under extremely harsh testing conditions. For these experiments, we observed that each reader was responsible for roughly $20 \%$ of the tags during the online phase, thus reflecting a reduction in computational load at each reader.

\section{F. Overlap Reduce Successive Cancelation Method}

The overlap reduced successive cancelation method relies on iterative piecewise elimination of tag correlations to achieve improved detection efficiency. This improved efficiency in detection may produce an undesired loss in detection accuracy. This simulation aims to show the tradeoff that can be achieved between improved computation and detection accuracy with the use of OR-SC. Fig. 16 plots the ratio of the number of correctly detected tags as we progress through the blocks of computation. The experiment is run with a set of 100 nodes over a transmission burst of $10^{4} \mu \mathrm{s}$ in a plane with $d_{\max } / d_{\min }=1$ and SNR at $15 \mathrm{~dB}$. We divide the received signal into 12 blocks, and compare the performance with increased 


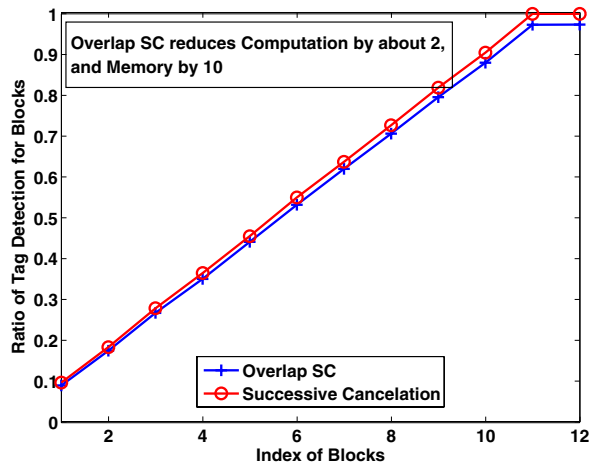

Fig. 16. Comparison Between Overlap Reduce Successive Cancelation Method and Successive Cancelation Method, with $d_{\max } / d_{\min }=$ $1, N=100$ and SNR is $15 \mathrm{~dB}$.

number of blocks. It can be seen that as we progress with the experiment a small residual error begins to accumulate. This error can be attributed to the wrong estimation of some tags in the initial block which leads to a wrong estimation of other tags in the consecutive blocks.

\section{CONCLUSION}

In this paper, we have proposed new methods to improve the tracking of receiverless transmit-only RFID tags. In a receiverless transmit-only RFID system, it is not possible to perform carrier sensing or collision avoidance and thus the challenge lies in resolving tag collisions. Our basic approach to address this problem is to utilize an enhanced form of multiuser detection at the receiver that can identify overlapping tag signals. We have developed a statistical algorithm to estimate signal amplitude and transmission time that exploits the properties of our tag system, and have integrated these algorithms to achieve an improved successive cancelation algorithm. Our successive cancelation method shows a better performance than the traditional successive cancelation method. Further, by making use of soft tag handoff between tag readers, and updating local tag lists at each reader, we have balanced the computational load across the entire system for improved scalability. In addition, we proposed a new overlap reduced successive cancelation method to further reduce the intensive computation and memory costs associated with successive cancelation. The performance under different collision situations, varying levels of near far effects, and noise are examined in simulations, and it is shown that our approach can reliably detect a large number of tags in a realistic inventory-monitoring scenario.

\section{REFERENCES}

[1] A. Juels. Rfid security and privacy: a research survey. $S e$ lected Areas in Communications, IEEE Journal on, 24(2):456460, April 2006.
[2] R. Weinstein. Rfid: A technical overview and its application to the enterprise. In IEEE Computer Technology, pages 27-33, May 2005.

[3] G. Roussos. Enabling RFID in retail. In IEEE Computer Technology, pages 27-33, March 2006.

[4] G. Bhanage, Y. Zhang, Y.Y. Zhang, W. Trappe, and R. Howard. Rollcall: The design for a low-power and highly-robust asset tracking system. Technical report, Technical Report WINLABTR-291, WINLAB, Rutgers University, December 2006.

[5] A. Jones, R. Hoare, S. Dontharaju, and S. Tung. An automated,reconfigurable, low-power RFID tag. In Design Automation Conference, 2006 43rd ACM/IEEE, 2006.

[6] S. Jain and S. Das. Collision avoidance in a dense RFID network. In WiNTECH, pages 49-56, September 2006.

[7] F. Schoute. Dynamic frame length ALOHA. In IEEE Transactions on Communications, pages 565-568, April 1983.

[8] M. Kodialam and T. Nandagopal. Fast and reliable estimation schemes in rfid systems. In MobiCom, pages 322-333, September 2006.

[9] B. Zhen, M. Kobayashi, and M. Shimizu. To read transmitteronly rfid tags with confidence. In PIMRC'04, September 2004.

[10] J. Cha and J. Kim. Novel anti-collision algorithms for fast object identification in rfid system. In Proceedings of the 11th International Conference on Parallel and Distributed Systems - Workshops (ICPADS'05), pages 63-67, September 2005.

[11] C. Floerkemeier and M. Wille. Comparison of transmission schemes for framed aloha based rfid protocols. In Applications and the Internet Workshops, International Symposium on, January 2006.

[12] D. Hush and C. Wood. Analysis of tree algorithms for rfid arbitration. In IEEE International Symposium on Information Theory, August 1998.

[13] C. Law, K. Lee, and K. Siu. Efficient memoryless protocol for tag identification. In Proceedings of the 4th International Workshop on Discrete Algorithms and Methods for Mobile Computing and Communications, August 2000.

[14] F. Zhou, C. Chen, D. Jin, C. Huang, and H. Min. Evaluating and optimizing power consumption of anti-collision protocols for applications in RFID systems. In ISLPED'04, August 2004.

[15] J. Myung and W. Lee. An adaptive memoryless tag anti-collision protocol for RFID networks. In IEEE ICC, March 2005.

[16] H. Vogt. Efficient object identification with passive RFID tags. In International Conference on Pervasive Computing, pages 98113, 2002.

[17] S. Verdu. Multiuser Detection. Cambridge University Press, Pitt Building, Trumpington Str., Cambridge, UK, first edition, 1998.

[18] J. Proakis. Digital Communications. McGraw-Hill Companies, Inc., fourth edition, 2001.

[19] A. Almutairi, S. Miller, H. Latchman, and T. Wong. Power control algorithm for mmse receiver based cdma systems. In Communications Letters, IEEE, pages 346-348, November 2000.

[20] N. Benvenuto, B. Carnevale, and S. Tomasin. Energy optimization of cdma transceivers using successive interference cancellation. In Globecom, pages 2644-2648, 2004.

[21] J.G. Andrews and T.H.Y. Meng. Amplitude and phase estimation considerations for asynchronous cdma with successive interference cancellation. In VTC, pages 1211-1215, 2000.

[22] P. Patel and J. Holtzman. Analysis of a ds/cdma successive interference cancellation scheme using correlations. In Globecom, pages 76-80, 1993.

[23] A. Goldsmith. Wireless Communications. Cambridge University Press, Stanford University, 2004. 